Running head: AGING AND NON-LITERAL LANGUAGE

\title{
Age-related differences in the ability to decode intentions from non-literal language
}

\author{
Christina Pomareda ${ }^{\mathrm{a}, 1}$, Auste Simkute ${ }^{\mathrm{a}}$ \& Louise H. Phillips ${ }^{\mathrm{a}}$
}

${ }^{a}$ School of Psychology, University of Aberdeen, Scotland, UK

Correspondence regarding this article should be addressed to Louise H. Phillips, Email address: louise.phillips@abdn.ac.uk. School of Psychology, University of Aberdeen, Aberdeen, Scotland, UK, AB24 3FX.

Other authors' Email addresses:

Christina Pomareda: cp670@cam.ac.uk

Auste Simkute: a.simkute.15@aberdeen.ac.uk.

Declaration of interests: none.

${ }^{1}$ Present address: Department of Psychology, University of Cambridge, England, UK 


\begin{abstract}
Older adults sometimes experience difficulty in decoding non-literal language, such as sarcastic statements where the underlying meaning differs from the literal words used. Given that sarcasm usually communicates a negative message this age effect might be explained by a positivity bias in old age. Here we test this for the first time by looking at age differences in interpreting non-literal compliments made with positive intention. However, another possibility is that older adults may fail to interpret such remarks correctly because these nonliteral compliments are rarely encountered in everyday interactions. The aim of this study was therefore to compare younger and older adults' comprehension of positively and negatively valenced non-literal language. Forty younger and thirty-eight older adults read short story scenarios ending with a positive or negative, literal or non-literal evaluative appraisal of an event. Older adults were less likely than young to detect negatively valenced non-literal criticism and there were even more pronounced age-related differences in comprehending positive non-literal compliments. This indicates that age differences in understanding nonliteral language are not driven by positivity biases. The relative rarity of non-literal compliments may have made these particularly difficult to interpret for both younger and older adults. Younger adults' performance indicated that non-literal language mutes perceived levels of critique and praise, while older adults' tendency to misinterpret non-literal language means that they may not benefit from this muting function. Potential implications for social interactions in older adulthood are discussed.
\end{abstract}




\section{Highlights:}

- Older adults were less likely than young to correctly interpret non-literal language.

- There was evidence against the role of positivity biases in these age effects.

- Instead age differences were stronger on more unusual linguistic forms.

- Older and younger adults interpreted non-literal compliments with more difficulty relative to typical sarcasm.

- Older adults may lose the benefits of communicating with non-literal speech.

Keywords: non-literal language, old age, mental state attribution, positivity bias, sarcasm

Classification codes:

2340 Cognitive Processes

2860 Gerontology

2750 Linguistics \& Language \& Speech 


\section{Introduction}

The meaning people intend to convey when they speak often goes beyond what is literally said. For example, a person may say "I see you're on time as usual!" when a colleague turns up late: a sarcastic statement where the literal meaning is positive, but the underlying intent is to convey a negative, critical meaning. Producing and comprehending non-literal language is more cognitively demanding than using or understanding literal language (Filik, Brightman, Gathercole, \& Leuthold, 2017; Dews, Kaplan, \& Winner, 1995). However, it has been suggested that non-literal language may fulfill unique socio-emotional functions such as strengthening social bonds or reducing the social cost of criticising others (Dews \& Winner, 1995; Filik et al., 2016). Theory of Mind (ToM), the ability to understand others' mental states (Premack \& Woodruff, 1978) is important for making non-literal interpretations of speech, and ToM is known to decline in old age (Henry, Phillips, Ruffman, \& Bailey, 2013; Moran, 2013). Indeed, older adults have specific difficulties in interpreting non-literal meanings from sarcastic statements (Phillips et al., 2015).

However, so far, no study has explored the impact of age on comprehension of positively valenced non-literal language (for example, uttering "You're a terrible cook!" when in fact the food was clearly very nice (see Table 1): i.e. using a joke 'criticism' to convey a compliment. Exploring this issue is interesting given both supportive evidence for (1) the presence of age-related positivity biases in interpreting social information (e.g., Reed, Chan, \& Mikels, 2014) and (2) the finding that non-literal criticism (e.g. sarcasm) is generally easier to understand than positively valenced non-literal compliments, as the latter are relatively rare in everyday speech (Giora, 1997). In the current study we explore the effects of aging on interpreting positive and negative non-literal statements to test whether older adults are more likely to place a positive spin on all potentially ambiguous utterances (in line with 
positivity biases), or instead fail to see the positive meaning behind non-literal compliments (because these are more unfamiliar and require more cognitive resource).

Understanding the meaning that is conveyed in a message requires the consideration of both the message itself (semantics and syntax) as well as the social context in which it is embedded (pragmatics, Boylan \& Katz, 2013). Pragmatics refer to how language is interpreted rather than linguistically decoded (Langdon, Davies, \& Coltheart, 2002). Dialogue partners sometimes communicate their beliefs and intentions indirectly via figurative or nonliteral language (e.g., via hyperboles) in everyday interactions (Roberts \& Kreuz, 1994). Two common forms of non-literal language, negatively valenced sarcasm and positively valenced irony (see Table 1), share the characteristic of involving a statement that is often opposite to an intended meaning (Pexman \& Olineck, 2002).

The terminology used to describe these two linguistic phenomena varies considerably across the literature. For clarity, here we use the terms non-literal criticism, which includes the use of sarcasm, and occurs when the intention is to criticise but the literal meaning of a statement is positive. In contrast, non-literal compliments occur when the intention is positive but the literal meaning is negative.

Table 1. Examples of non-literal criticism and compliment

\begin{tabular}{cccc}
\hline Terminology & $\begin{array}{c}\text { Literal } \\
\text { meaning }\end{array}$ & Intention & Example \\
\hline Non-literal criticism & Positive & Negative & Uttering "You're a brilliant cook" when \\
('blame by praise') & & & in fact the food was horrible. \\
Non-literal compliment & Negative & Positive & Uttering "You're a terrible cook" when \\
('praise by blame') & & & in fact the food was very nice. \\
& & & \\
\hline
\end{tabular}


It is important to note that the goal of using non-literal language is not to deceive. Rather, the addressee is expected to recognise and appreciate the disparities in stated and intended meanings (Bruntsch \& Ruch, 2017; Shamay-Tsoory, Tomer, \& Aharon-Peretz, 2005). Several factors have been identified to aid successful interpretation: the situational context, such as an inconsistency between experiencing a delayed train and a positive appraisal of that situation (Kreuz \& Link, 2002), shared knowledge between the speaker and the listener based on past common experience (Shamay-Tsoory et al., 2005), or paralinguistic cues such as tone of voice and facial expression (Kreuz \& Roberts, 1995). Due to variations in availability and accessibility of such cues, comprehending non-literal language is often significantly more complex than understanding literal language (Filik et al., 2017). Thus, by speaking non-literally, the risk of being misunderstood increases. Yet, in spite of these associated costs, people often deliberately choose non-literal over sincere language. It has been demonstrated that non-literal language must, therefore, convey distinct information that literal language cannot convey in the same way (e.g., Dews \& Winner, 1995; Colston, 1997; Bruntsch \& Ruch, 2017; Filik et al., 2016, 2017).

An important social function of non-literal language might be to attenuate or mute the negative valence of a message underlying a statement. According to the Tinge Hypothesis (Dews \& Winner, 1995), non-literal criticisms are perceived as less mean than literal criticisms and non-literal compliments appear to be less positive than literal compliments. To illustrate, if a burnt meal was sarcastically described as looking "brilliant" (e.g., non-literal criticism), the literally positive valence of the adjective "brilliant" would tinge perception so that the remark would be perceived as less hurtful compared to using a literally negative word such as "horrible" instead. Non-literal criticism can serve a face-saving function and protect the speaker from transgressing social norms (Glucksberg, 1995; Dews et al., 1995). For 
example, Boylan and Katz (2013) report that non-literal criticism was rated as more mocking but also more polite than direct criticism. Non-literal criticism (e.g., saying "Well done!" when someone makes a bad mistake) might also serve to reduce threat (Brown \& Levinson, 1987), strengthen social bonds or create humorous effects (Dews \& Winner, 1995). Similarly, conveying compliments non-literally (e.g. saying "You are such a terrible cook!", following a meal that everyone clearly enjoyed a lot) can protect the speaker from losing status and reduce the risk of embarrassing the addressee (Pexman \& Olineck, 2002). In the case of nonliteral compliments (e.g., saying "You're a slow coach!" when someone has just won a race), the negatively connotated adjective "slow" would make the compliment appear less positive. Non-literal compliments can serve to entertain, flirt, be funny or witty, or remind the addressee of social norms in a playful way (Bruntsch \& Ruch, 2017; Roberts \& Kreuz, 1994).

Understanding of non-literal language develops across the lifespan. Children only gradually develop the pragmatic skills to interpret non-literal messages (Sullivan, Winner, \& Hopfield, 1995; Winner \& Leekam, 1991). In particular, understanding of non-literal compliments develops late, possibly because this type of language is encountered relatively infrequently (Hancock, Dunham, \& Purdy, 2000; Harris \& Pexman, 2003). There is little known about adult lifespan development and interpreting non-literal criticism and compliments. Given age-related declines in the ability to interpret others' mental states (Henry et al., 2013), and emotions (Ruffman, Henry, Livingstone, \& Phillips, 2008) agerelated problems might also be predicted in detecting negative (Phillips et al., 2015) or positive intentions from non-literal language.

In line with this possibility that older adults might misinterpret non-literal language there is evidence that older adults are less able to process some aspects of humour (Uekermann, Channon, \& Daum, 2006) as well as proverbs and metaphors (Uekermann, Thoma, \& Daum, 2008). While generating and understanding proverbs and metaphors is 
complex, these forms of figurative language may help to express discourse goals which could not be conveyed as effectively via literal language (Kreuz \& Link, 2002). In contrast, Champagne, Seendy and Joanette (2006) suggest that age differences in understanding nonliteral language may be minimal once differences in education are accounted for.

Few studies have directly looked at the effects of adult aging on understanding nonliteral criticism (e.g., Champagne et al., 2006; Phillips et al., 2015), and none have looked at age differences in interpreting non-literal compliments. The ability to comprehend sarcasm and irony is associated with unique socio-emotional functions (e.g., Dews et al., 1995; Filik et al., 2016, 2017), which may be highly relevant for older adults' social functioning. Phillips et al. (2015) looked at the effects of aging on understanding of sarcasm (i.e. non-literal criticism). They found that older adults, compared to both younger and middle-aged participants, were significantly worse at understanding both written and video-taped sarcastic exchanges. There were no age differences in identifying literal statements. Facial emotion perception skills mediated age effects in interpreting sarcasm from video clips, whereas age differences could not be explained in terms of working memory.

One important factor which might have influenced the results of the Phillips et al.'s (2015) study is that all of the sarcastic statements were non-literal criticisms: in other words, the literal meaning (e.g. "That was a fantastic play you took me to see") was positive but the underlying meaning was negative (e.g. "The play was terrible"). This is problematic because of the age-related positivity bias. Older adults are more likely to ignore negative information and attend to positive information compared to young (e.g., Charles \& Carstensen, 2008). This might mean that older people are more likely to interpret contradictory or conflicting information such as non-literal statements in the direction that is most positive. This would fit with a pattern whereby older adults interpreted sarcastic statements (e.g., non-literal criticisms) in line with the surface positive meaning rather than underlying negative meaning. 
In order to investigate this issue in the current study we looked at age differences in interpreting non-literal language which had either positive or negative underlying meaning using stories constructed to portray non-literal criticism (underlying meaning negative but surface meaning positive) and non-literal compliments (underlying meaning positive but surface meaning negative), see Table 1 . If positivity biases were an important influence on the way that older adults interpret language then we would expect that older adults would misinterpret non-literal criticism (sarcasm) in line with the surface meaning, whereas they would correctly interpret non-literal compliments in line with the underlying positive meaning.

An alternate prediction can also be made. As non-literal criticism (typical sarcasm) is encountered more frequently than non-literal compliments, listeners are naturally less familiar with the latter (Kreuz \& Link, 2002), and may require more cognitive resources to understand their meaning. Giora (1997) put forward the 'graded salience' hypothesis, which proposes that the more familiar the structure of a speech utterance, the more it will be processed automatically. Given that non-literal compliments are rare in everyday speech it can be predicted that older adults (as well as younger adults) may therefore be particularly likely to misinterpret non-literal compliments in line with the (negative) surface meaning as opposed to the (positive) underlying meaning because these types of remarks are relatively rare and require higher levels of controlled cognitive processing.

In the current study we therefore looked at the effects of adult aging on interpreting non-literal remarks with different emotional connotations. Participants were shown short vignettes ending in a remark which could be literal or non-literal, with an underlying negative or positive intention. They rated the degree to which the intentions of the protagonist were kind or mean, and we also assessed their understanding of the mental state of the protagonist. If positivity biases underlie previous evidence of age-related problems in interpreting sarcasm 
(Phillips et al., 2015) then older adults will most likely interpret non-literal compliments in line with the positive (underlying) meaning rather than the negative (surface) meaning. In contrast, if processing non-literal compliments is more cognitively demanding because such constructions are unfamiliar and less automatically processed, older adults will tend to interpret them in line with the simpler (negative) surface meaning, rather than the underlying (positive) meaning.

In line with the findings of Phillips et al. (2015) we predicted that there would not be age differences in interpreting the meaning from literal statements. To further explore whether more general age-related difficulties in processing language might be important, understanding and memory of each storyline was assessed via a factual understanding question. As younger and middle-aged adults performed similarly in Phillips et al.'s (2015) study, an extreme age design consisting of younger and older adults only was used here.

\section{Method}

\subsection{Participants}

A total of 78 participants (20 males, 58 females) gave written informed consent to take part in the experiment: 40 younger adults ( 9 males, 31 females) ranged in age from 18 to 25 years $(M=21.15, S D=1.89)$, and 38 older adults (11 males, 27 females $)$ were aged between 65 and 79 years $(M=70.87, S D=4.14)$. The older participants were recruited through the participant panel in the School of Psychology and were reimbursed $£ 12$ for their time. All younger adults were recruited via word of mouth or through the School of Psychology internal Research Participant Scheme and were mostly undergraduate students participating in exchange for course credit. All participants self-rated their health on a scale from $1-9$ (e.g., 1 = very poor, 9 = very good) and indicated how many years of education they had completed to date. There was no significant difference in self-rated health between 
age groups, $t(76)=1.78, p=.070$ (younger adult $M=7.70, S D=1.11$; older adult $M=7.26$, $S D=0.98$ ), and no significant difference in years of education between age groups, $t(56.38)=-1.24, p=.219$ (younger adult $M=14.35$ years, $S D=1.55 ;$ older adult $M=15.0$ years, $S D=2.9$ ). No participant reported current or past neurological or psychiatric problems, except for one older adult with a recent mild stroke. This participant was not excluded from the study as there were no residual deficits, and they were not an outlier on any of the measures. All participants were English-speaking. All older participants except for one were native English speakers. All younger non-native English speakers (60\% of the younger sample) were currently enrolled in Scottish full-time higher education. The study received ethical approval from the Psychology Ethics Committee at the University of Aberdeen.

\subsection{Materials}

All participants completed the 20-item Positive and Negative Affect Schedule (PANAS-20, Watson, Clark \& Tellegen, 1988) at the start of the session to assess how positive (younger adult $M=29.88, S D=5.87$; older adult $M=34.34, S D=6.74 ; t(76)=-$ $3.13, p=.003, d=2.13$ ) and negative (younger adult $M=14.45, S D=5.21$; older adult $M=$ $11.18, S D=2.02 ; t(76)=3.6, p=.001, d=0.83)$ they felt at that point in time. After the literal and non-literal Stories Task to test comprehension of intentions (described in detail below), additional questionnaires were administered as part of another unrelated study which most participants also completed.

\subsection{Stimuli: Stories intention comprehension task:}

Short stories describing an interaction between two people were developed (see Table 2). Story themes were selected to be accessible and relevant to both younger and older participants. There were four different story sets developed. Set 1 (1i-1viii) and 2 (2i to 2viii) each consisted of 8 different story themes (e.g., trying to play golf for the first time). Stories 
belonging to sets 3 (3i-3viii) and 4 (4i-4viii) were structurally and thematically matched to the stories in group 1 and 2, respectively, but differed in terms of the story characters' names and activities described (e.g., John playing golf in story 1ii was modified to Jack playing tennis in story 3ii). This made 32 distinguishable story types in total. Each of these 32 story types had four conditions (literal compliment, non-literal compliment, literal criticism, nonliteral criticism). The four conditions corresponding to the same story type (e.g., John playing golf in 1ii) were structurally highly similar, with variations in contextual information and content of the final statements to give cues to whether the statement was meant literally or not, see Table 2 for an example. In total, there were therefore 128 possible stories.

Two parallel versions of the Stories Task (A and B) were generated for the final experiment, with 32 stories in each. The stories used in version A and B were closely matched for length, syntactic structure, themes and the characters' gender in order to control for item variability. Half of the participants of each age group were tested on version A and the other half on version B. Both versions were split into block 1 and block 2, each containing 16 stories. Block 1 of version A contained one condition from each of the stories in sets 1 and 2, selected so that there were four examples of each condition (literal compliment, non-literal compliment, literal criticism, non-literal criticism) in the whole block. The second block of version A consisted of one condition from each of the stories in sets 3 and 4, again selected so that there were four examples of each condition. Exactly the same structure was used for version $\mathrm{B}$, but with different choices of condition for each story set. This meant that no story presented to participants in version A was the exact same as presented to participants completing version B, and no participant read different conditions of the same story (i.e. if they read Story $1 \mathrm{ii}$ in the literal compliment condition they would not see the same story in any of the other three conditions). Note that analysis confirmed that there was no effect of 
AGING AND NON-LITERAL LANGUAGE

version $(\mathrm{A} / \mathrm{B})$ on performance so this is not further considered here. The full list of story materials used in the experimental task is presented in Supplementary Materials 1. 
Table 2. Example of the experimental material used for the Stories Task. Each participant saw only one of the four possible sub-conditions from any story theme. Cue words were not made salient in the experimental stimuli presented to participants.

\begin{tabular}{|c|c|c|}
\hline Literality & $\begin{array}{l}\text { Valence of } \\
\text { intention }\end{array}$ & Example scenario (emphasis added) \\
\hline Literal & Compliment & $\begin{array}{l}\text { John had always been pretty good at sports but he hadn't tried } \\
\text { golf before. After trying it out for the first time, he went home } \\
\text { and told his twin brother George that he really enjoyed trying it } \\
\text { out. George raised his eyebrows and said "I bet you were great } \\
\text { at it, like most sports". }\end{array}$ \\
\hline & Criticism & $\begin{array}{l}\text { John had never really been very good at sports but he hadn't } \\
\text { tried golf before. After trying it out for the first time, he went } \\
\text { home and told his twin brother George that he really enjoyed } \\
\text { trying it out. George replied with a chuckle "I bet you were } \\
\text { rubbish at it, like most sports". }\end{array}$ \\
\hline
\end{tabular}

Non-literal Compliment John had always been pretty good at sports but he hadn't tried golf before. After trying it out for the first time, he went home and told his twin brother George that he really enjoyed trying it out. George replied with a chuckle "I bet you were rubbish at it, like most sports".

Criticism John had never really been very good at sports but he hadn't tried golf before. After trying it out for the first time, he went home and told his twin brother George that he really enjoyed trying it out. George raised his eyebrows and said "I bet you were great at it, like most sports". 
Each short story scenario featured a social interaction between two people, always ending with one person (the speaker) making a final utterance (the target statement) towards his or her dialogue partner (the addressee). The target statements always contained a positive or negative appraisal of a previously described circumstance. The contextual information preceding the target statement supported the interpretation of the speaker's intention as sincere or non-literal.

\subsection{Procedure: Stories comprehension task}

Before proceeding to the 32 main short story trials, a practice trial allowing participants to familiarize themselves with the task requirements was completed. The stories were presented in text format on a computer screen. The time participants could take to read a story was not restricted. This design was employed to avoid experimentally manipulating reading time, given age-related differences in reading speed (Hartley, Stojack, Mushaney, Annon, \& Lee, 1994) and the differentially high processing demands of literal versus nonliteral story conditions (Filik et al., 2016). After reading each story, participants had to rate the degree to which they thought the speaker's final utterance was intended to be kind or mean on a scale from $1-9(1=$ 'extremely mean', $9=$ 'extremely kind'). This differentiated scale accounted for the ambiguity of the scenarios by leaving room for fine nuances in the rating. Next, participants answered a mental state question (MSQ) assessing awareness of whether the intention conveyed in the speaker's final utterance was sincere or not (e.g., "Does George believe John is good at golf?"). Participants' comprehension of the basic storyline was also assessed via a factual understanding question (FUQ) (e.g., "Is John usually good at sports?").

The order in which the questions appeared (MSQ or FUQ first) was counterbalanced across version A and B. FUQs and MSQs required a simple 'yes' or 'no' response each, indicated by pressing the ' $y$ ' or ' $n$ ' key on the keyboard, respectively. Participants were not 
able to go back to the story once they had proceeded to the rating scale and the questions. This design allowed for a more realistic simulation of real-life social interactions, which would not allow for spoken or written statements (e.g., in an online context) to be rehearsed multiple times before a response has to be made. New trials were self-initiated by pressing the space key button on the keyboard. Halfway through the task, after having completed 16 stories in block 1, participants were free to take a break for as long as they wished. Within blocks 1 and 2 the order of presentation of the stories was randomised. Once the experimental session was finished, participants were monetarily reimbursed or allocated course credit for taking part and were verbally debriefed by the experimenter.

\subsection{Design and analysis.}

The study was a 2 (age group: older vs. younger) x 2 (literality: literal vs. non-literal) x 2 (valence: criticism vs. compliment) design with age group as a between-subjects factor and literality and valence of the stories as within-subjects factors. Separate 2 (older vs. younger) x 2 (literal vs. non-literal) x 2 (criticism vs. compliment) mixed design analyses of variance (ANOVAs) were conducted on the dependent variables of (a) the number of correctly answered mental state questions, (b) the kindness ratings, and (c) the number of correctly answered factual understanding questions. Where interactions with age were significant, paired samples t-tests comparing age groups on each condition were carried out. The stories used in the current study were systematically matched to minimise variability within and across story conditions (see sections 2.3 and 2.4 for details). Traditional (F1) analyses were run with subjects as random factors and items as fixed factors following advice from Raaijmakers, Schrijnemakers and Gremmen (1999) that item analyses are not needed when the items are appropriately matched and counterbalanced. 


\section{Results}

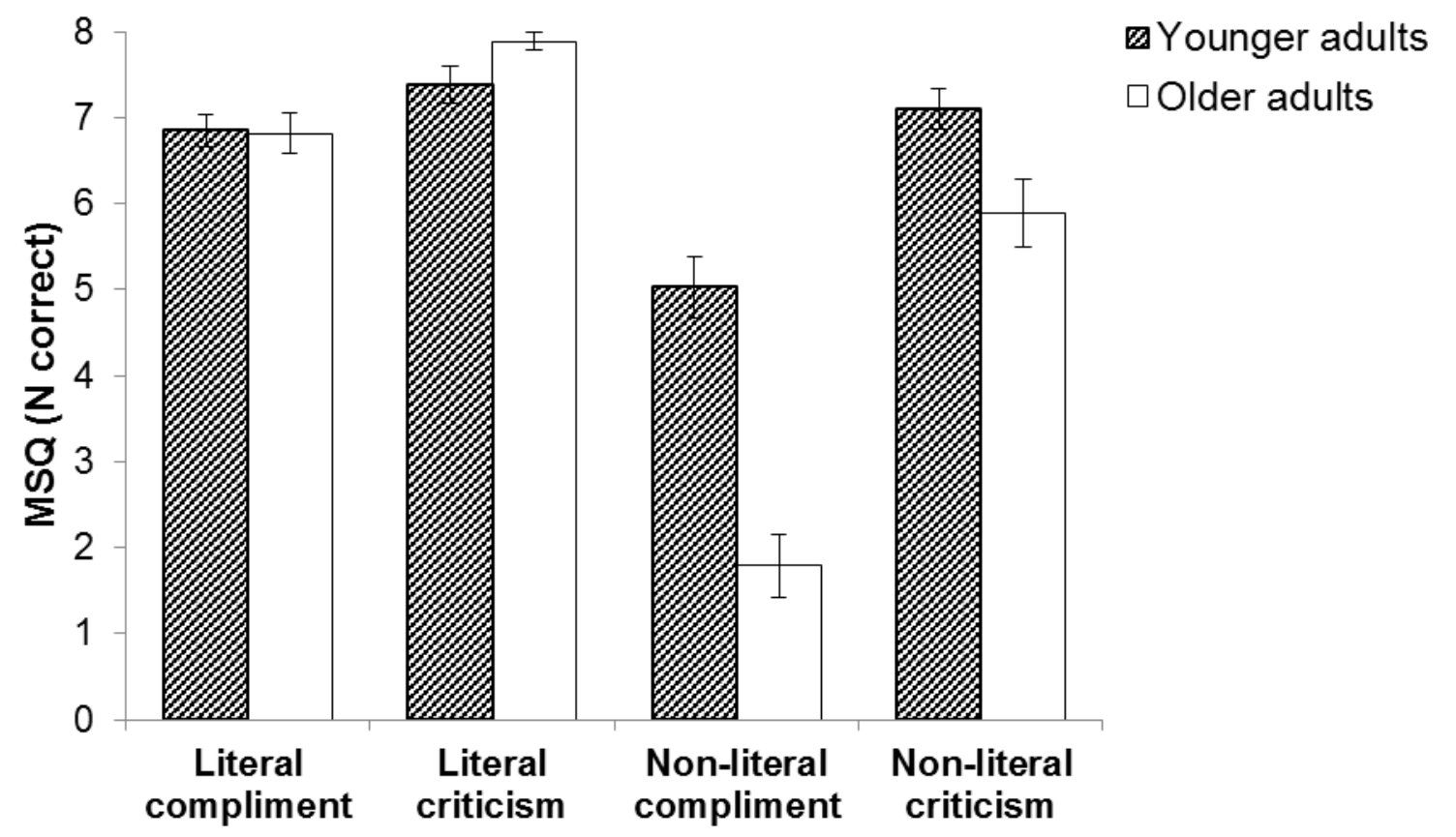

Figure 1. Mean number of mental state questions (MSQs) answered correctly across story conditions and age groups. Error bars indicate \pm 1 SEM.

3.1 Mental state questions: A mixed-design ANOVA on mental state understanding revealed a significant main effect of literality, $F(1,76)=101.23, p<.001, \eta p^{2}=.57$, with literal questions being answered more accurately than non-literal. There was also a main effect of valence, $F(1,76)=126.88, p<.001, \eta p^{2}=.63$, because compliments were interpreted less accurately than criticisms. There was a significant main effect of age group on the number of correctly answered mental state questions, $F(1,76)=22.71, p<.001, \eta p^{2}=$ .23. Younger adults $(M=6.59)$ answered significantly more mental state questions correctly than older adults $(M=5.60)$.

There were significant interactions between valence $\mathrm{x}$ literality, $F(1,76)=63.71, p<$ $.001, \eta p^{2}=.46$, age group and literality, $F(1,76)=29.50, p<.001, \eta p^{2}=.28$; and between 
age group and valence, $F(1,76)=13.98, p<.001, \eta p^{2}=.16$; but these were superseded by a significant three way interaction between age, valence and literality, $F(1,76)=6.63, p=.012$, $\eta p^{2}=.08$, see Figure 1 . To decompose this three-way interaction, paired-samples t-tests were run and revealed that older adults were less accurate in attributing mental states from nonliteral criticism $(M=6.00)$ compared to younger adults $(M=7.10), t(57.97)=2.69, p=.009$, $d=0.63$. An even larger age difference was found in interpreting mental states from nonliteral compliments (older adult $M=1.79$, younger adult $M=5.03, t(74)=6.04, p<.001, d=$ 1.39). In contrast, older adults were more accurate than young in interpreting mental states in stories containing literal criticism (older adult $M=7.90$; younger adult $M=7.38, t(43.65)=-$ 2.30, $p=.026, d=0.52$ ). There was no age difference on literal compliment trials (older adult $M=6.82$; younger adult $M=6.85, p=.881)$. The results indicate that older adults had difficulty decoding mental states linked to both positive and negative non-literal remarks, but not with understanding those linked to sincere comments. The age effect size for interpreting non-literal compliments was more than twice as big as for non-literal criticisms.

\subsection{Mean kindness rating assessment: A mixed-design ANOVA on mean kindness} ratings revealed a large main effect of literality, $F(1,76)=70.70, p<.001, \eta p^{2}=.48$, (literal statements rated as more kind than non-literal) and valence, $F(1,76)=92.85, p<.001, \eta p^{2}=$ .55 , (compliments rated as more kind than criticisms). There was a significant main effect of age on mean kindness ratings, $F(1,76)=5.30, p=.024, \eta p^{2}=.07$. Older adults $(M=4.82)$ rated the stories as significantly less kind than younger adults $(M=5.20)$. 


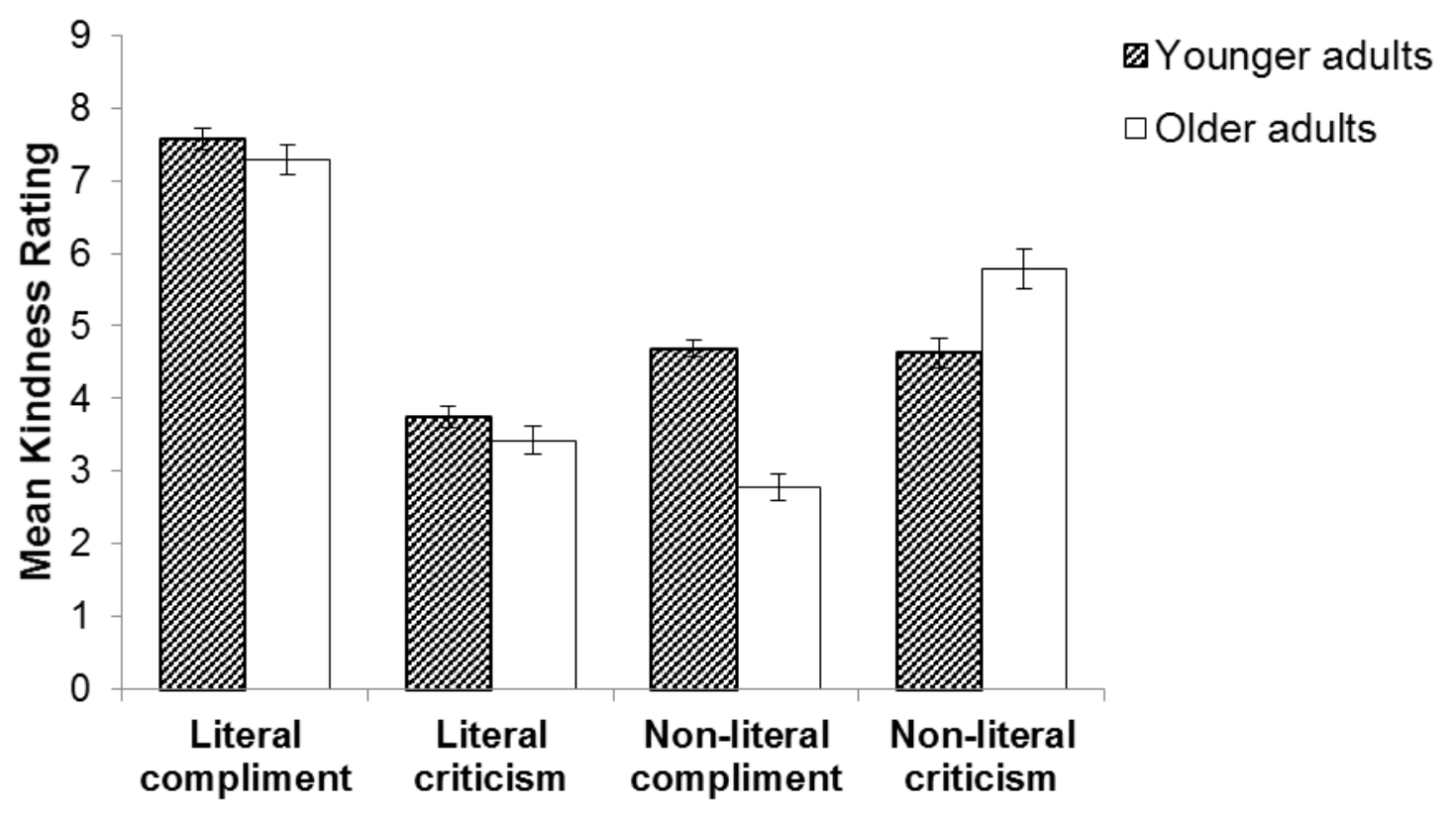

Figure 2. Mean kindness rating score across age groups and story conditions. Error bars indicate $\pm 1 S E M$.

There was no interaction between age and literality, $F(1,76)=0.10, p=.756, \eta p^{2}=$ .001 , but a significant interaction between literality and valence, $F(1,76)=270.53, p<.001$, $\eta p^{2}=.78$; and age and valence, $F(1,76)=38.59, p<.001, \eta p^{2}=.34$, and a significant three way interaction between age, literality and valence, $F(1,76)=22.99, p<.001, \eta p^{2}=.23$, see Figure 2. Paired-samples t-tests revealed no age difference in kindness ratings for either literal compliments, $t(65.57)=1.14, p=.259$, or literal criticisms, $t(76)=1.33, p=.188$. Non-literal criticisms were rated as significantly more kind by older adults, $t(68.47)=-3.43$, $p=.001, d=-0.78$, while in contrast non-literal compliments were rated as more kind by younger participants, $t(76)=6.76, p<.001, d=1.53$. These results indicate that older adults would be more likely than young to rate both types of non-literal exchanges to match the literal rather than intended meaning. Again, it can be noted that the age effect size was 
approximately twice as high for interpreting non-literal compliments compared to non-literal criticisms.

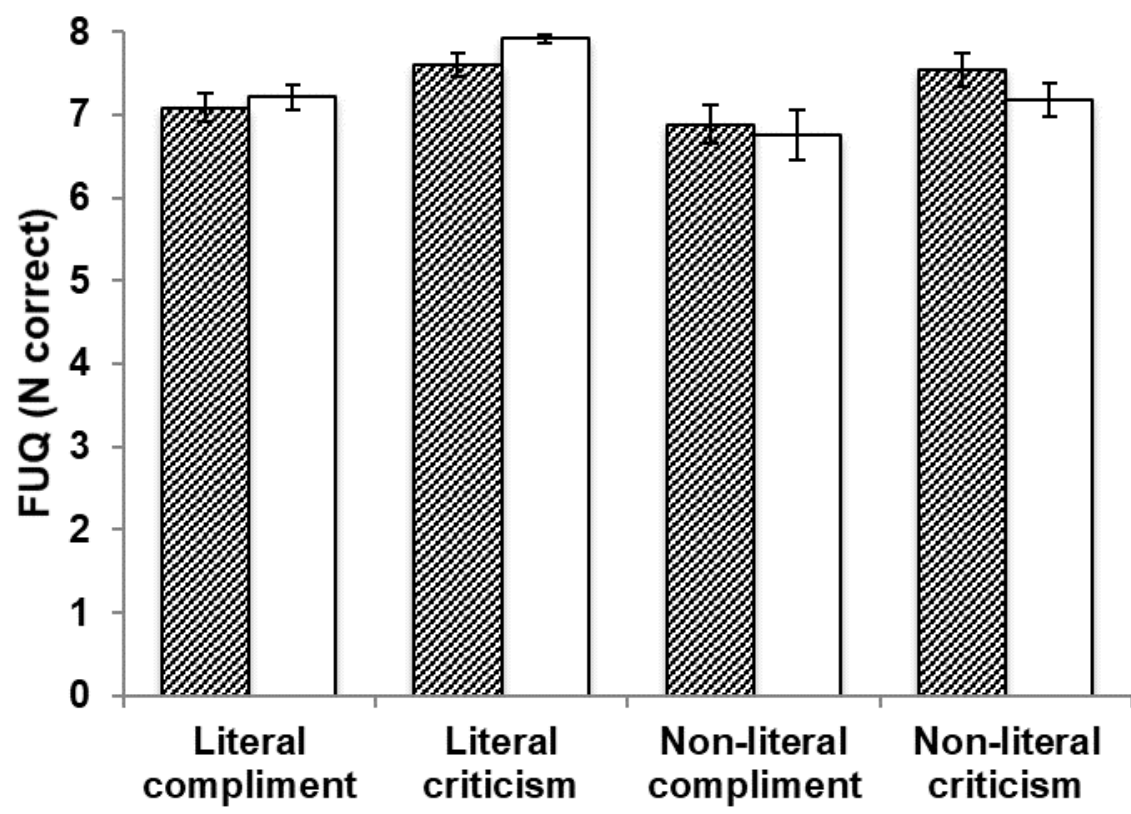

vYounger adults 口Older adults

Figure 3. Mean number of correctly answered factual understanding questions (FUQs) across story conditions and age groups. Error bars indicate $\pm 1 S E M$.

3.3 Factual understanding assessment: For the factual understanding questions, a mixed-design ANOVA revealed a main effect of literality, $F(1,76)=7.37, p=.008, \eta p^{2}=$ .09 , with greater understanding of story context in the literal compared to non-literal conditions. There was also an effect of valence, $F(1,76)=21.72, p<.001, \eta p^{2}=.22$, with greater understanding of stories containing criticism as opposed to compliments. There was no effect of age group on the number of correctly answered factual questions, $F(1,76)=$ $.001, p=.971, \eta p^{2}<.0$.

There was no literality $\mathrm{x}$ valence interaction, $F(1,76)=.09, \eta p^{2}<.01$, and no interaction between age group and literality, $F(1,76)=.3 .13, p=.081, \eta p^{2}=.04$, valence, $F$ $(1,76)=.02, p=.892, \eta p^{2}<.01$, or age $\mathrm{x}$ literality $\mathrm{x}$ valence, $F(1,76)=.84, p=.363, \eta p^{2}$ 
$=.01$. This indicated that older and younger adults' factual understanding of the context preceding the target statement did not differ.

\subsection{Correlations of Measures:}

To investigate how our measures of non-literal language comprehension, i.e., mean kindness ratings (MKRs) and mental state understanding (MSQ), related to each other, correlations between these measures for each story condition (e.g., literal compliment, literal criticism, non-literal compliment, non-literal criticism) were run. Results indicated significant positive correlations between success at MSQs and MKRs for literal $(r=.50, p<.01)$ and non-literal compliments $(r=.74, p<.01)$ indicating that greater success at inferring the speaker's mental state was associated with higher kindness ratings for literal and non-literal compliments. There were significant negative correlations between mental state comprehension and mean kindness ratings for stories containing either a literal $(r=-.34, p=$ $.002)$ or non-literal criticism $(r=-.50, p<.01)$, suggesting that correctly inferring the mental state of a speaker uttering a literal or non-literal criticism was related to perceiving the speaker's intention as less kind. These results show that both measures of intention understanding converge.

\subsection{Correlations of Measures with PANAS}

Table 3. Correlations of Positive and Negative Affect with Mean Kindness Ratings and Mental State Questions across Story Conditions.

\begin{tabular}{|c|c|c|c|c|c|c|c|c|}
\hline & \multicolumn{2}{|c|}{$\begin{array}{c}\text { Literal } \\
\text { Compliment }\end{array}$} & \multicolumn{2}{|c|}{$\begin{array}{c}\text { Literal } \\
\text { Criticism } \\
\end{array}$} & \multicolumn{2}{|c|}{$\begin{array}{c}\text { Non-literal } \\
\text { Compliment }\end{array}$} & \multicolumn{2}{|c|}{$\begin{array}{c}\text { Non-literal } \\
\text { Criticism }\end{array}$} \\
\hline & MKR & MSQ & MKR & MSQ & MKR & MSQ & MKR & MSQ \\
\hline PANAS-P & .12 & .04 & .05 & .05 & $-.39 * *$ & $-.31 * *$ & .12 & -.17 \\
\hline PANAS-N & -.03 & .12 & .03 & $-.23^{*}$ & .12 & .19 & -.02 & .17 \\
\hline
\end{tabular}

Note. $\mathrm{MKR}=$ mean kindness rating, $\mathrm{MSQ}=$ mental state question; PANAS-N and

PANAS-P = negative affect and positive affect score on the Positive and Negative Affect Schedule. 
$* p<.05 . * * p<.01$.

As outlined in the methods section, older adults had higher levels of positive affect and lower levels of negative affect compared to younger adults. To explore how differences in current affect might relate to performance on our measures of non-literal language comprehension, mean kindness ratings (MKRs) and mental state understanding (assessed via mental state questions; MSQs) were correlated with participants' levels of positive and negative affect, see Table 3. Results show that positive affect was significantly correlated with both lower success at inferring protagonists' mental states $(r=-.31)$ and lower mean kindness ratings $(r=-.39)$ for short stories containing a non-literal compliment. This indicates that participants who felt more positively at the time of completing the task were more likely to misinterpret non-literal compliments in line with their negative surface rather than intended positive meaning.

As age group was significantly negatively correlated with lower success at nonliteral compliment comprehension for both MKRs $(r=-.61, p<.001)$ and MSQs $(r=-.59, p$ $<.001)$ and age group was also significantly correlated with higher levels of positive affect $(r$ $=.34, p=.003$ ), we investigated whether positive affect statistically mediated the effects of age on non-literal compliment comprehension in the current study. 


\subsection{Mediation analysis}
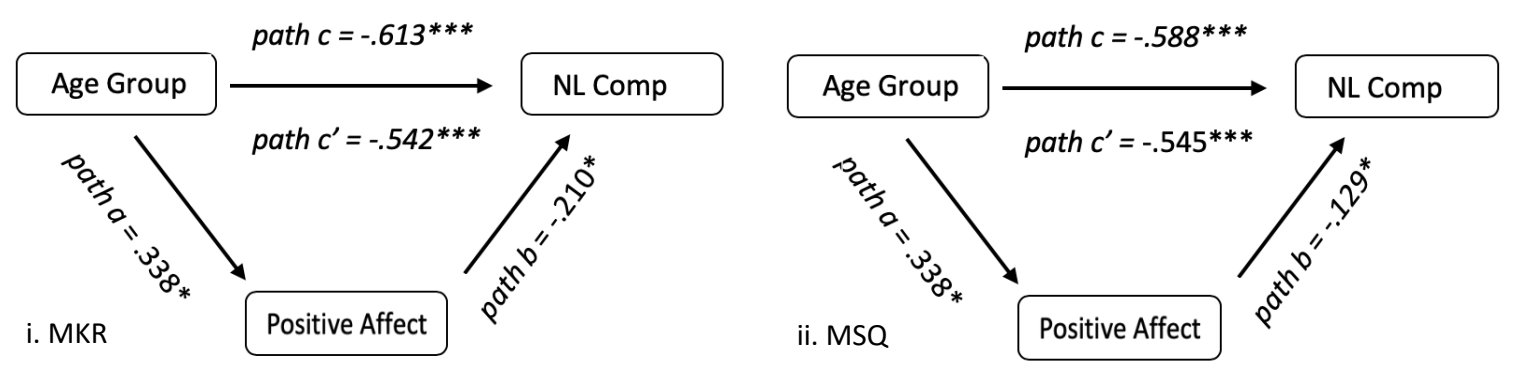

Figure 4. Path diagrams of mediation analyses examining positive affect as a potential mediator of age differences in non-literal compliment (NL Comp) comprehension for i. mean kindness ratings (MKRs) and ii. mental state questions (MSQS). Coefficient (c) indicates direct effects (e.g., age effect on non-literal language comprehension) and coefficient ( $\left.c^{\prime}\right)$ indicates indirect effects (e.g., age effect on non-literal language comprehension once positive affect is accounted for as a mediator in the model). Reductions from c to c' indicate a potential mediation effect.

$* p<.05 * * * p<.001$.

Following the methods outlined by Preacher and Hayes (2004), a series of linear regressions were conducted to test whether the correlation between the predictor (age group) and the outcome variable (non-literal compliment comprehension assessed via i. MKR or ii. MSQ) was significantly mediated by a third mediator variable (positive affect, PA). Bivariate regressions predicting the mediator variable (PA) from age group (paths $a$ in Figure 4) and predicting non-literal language comprehension from age group (paths $c$ in Figure 4) were carried out. A final multiple regression analysis was conducted to predict non-literal language comprehension from both the predictor (age group) and mediator (PA) together (paths $b$ and $c$ ' in Figure 4).

We first investigated the influence of PA on age group differences in kindness attributions to non-literal compliments (see Figure 4i). PA was a significant predictor of lower success at understanding non-literal compliments (e.g., lower mean kindness ratings) 
when entered along with age group in the analysis (see Figure 4i). There was a significant indirect effect of age on non-literal compliment comprehension through positive affect (bias corrected confidence intervals [-.442, -.013] do not include zero, see Figure 4i). Thus, positive affect significantly mediated the relationship between age and low mean kindness ratings for non-literal compliments. However, note that the relationship between age and nonliteral language remained highly significant after PA was factored in as a mediator (see Figure 4i). Second, the impact of PA on the relationship between age and lower success at inferring mental states from non-literal compliments (e.g., assessed via MSQs) was explored (see Figure 4ii). Bootstrapping revealed that PA did not significantly mediate the relationship between age and lower success at inferring mental states contained in non-literal compliments (confidence intervals [-.693 - .196] include zero, see Figure 4ii).

\section{Discussion}

This study was the first to investigate adult age differences in comprehension of negatively and positively valenced, literal and non-literal written exchanges. Overall, results supported the prediction that older adults, in contrast to their younger counterparts, exhibit specific difficulties in understanding non-literal language, with greater age differences for non-literal compared to literal understanding. These age differences extended to interpreting both positively and negatively intended non-literal remarks, which does not support the idea that previously reported age differences in understanding sarcasm (e.g. Phillips et al., 2015) might reflect a positivity bias in old age.

Older adults rated non-literal criticisms (e.g., saying "You're so clever!" when someone says something obvious) as significantly more kind and non-literal compliments (e.g., saying "The food is inedible!" when at the same time requesting a second portion) as significantly more mean than younger adults. In other words: older adults seemed more likely 
to interpret such remarks literally. Also, older adults answered significantly fewer mental state questions about both types of non-literal statements correctly than their younger counterparts. Both younger and older adults experienced more difficulty with interpreting non-literal compliments than non-literal criticisms. The age-effects were greatest for nonliteral compliment comprehension: older adults rated non-literal compliments as even more mean than literal criticisms. Also, older adults answered only 1.79 out of 8 mental state questions assessing the understanding of non-literal compliments correctly, indicating that they failed to understand the underlying intention behind these statements. In each condition, accuracy of understanding mental state questions was related in the expected direction to kindness ratings, indicating that both measures address the same construct.

The current research further extends Phillips et al.'s (2015) finding that older adults have difficulty understanding written sarcastic exchanges (non-literal criticism) by providing evidence for even more impaired non-literal compliment comprehension in older adults. In parallel fashion, developmental research indicates that $5-6$ years-old children more easily detect non-literal criticism than non-literal compliments (Hancock et al., 2000). Thus, this study shows for the first time that the relative asymmetry between ease of non-literal criticism versus compliment comprehension (e.g., Hancock et al., 2000) extends into older adulthood.

The current study therefore clearly provides evidence against the suggestion that positivity biases, i.e. older adults' preferential engagement with positive over negative social information (Reed et al., 2014; Luong \& Charles, 2014), may be key in explaining age differences in interpreting non-literal language. It has been suggested that older adults' positivity bias may mean that they are less alert to negatively valenced sarcasm than to more positively valenced forms of non-literal language (Phillips et al., 2015).Yet, although in the current sample older adults reported more positive affect than younger adults, older 
participants were particularly likely to interpret non-literal compliments to reflect the surface negative meaning rather than the underlying positive meaning.

Note that higher levels of positive affect were related to difficulties with interpretation of non-literal compliments for both younger and older adults. Thus, higher positive affect predicted a more negative interpretation of these remarks. We therefore explored the potential role of positive affect in the observed age differences in non-literal compliment comprehension via mediation analyses. Although positive affect partially mediated the relationship between age and lower kindness ratings for non-literal compliments, this only explained a small proportion of the age effect. Also, no mediation effect was observed on mental state questions. These results provide a hint that older adults in a more positive mood state may have been particularly likely to go along with the surface meaning of a remark. The fact that these relationships were only seen for non-literal compliments may reflect the more cognitively demanding nature of those constructions. However, these results should be interpreted tentatively, and explored in future research, perhaps with mood manipulations.

The finding that non-literal compliments were the most difficult to process is in line with predictions from the Graded Salience Hypothesis (Giora, 1997), which postulates that for relatively infrequently occurring non-literal compliments the level of controlled processing required is high. In contrast, as typical sarcasm is more frequently encountered and consequently more familiar or prototypical than non-literal compliments (Kreuz \& Link, 2002), the cognitive demands of understanding sarcasm may be more similar to the processing demands of literal language.

In contrast to our findings on non-literal statement interpretation, older and younger adults did not differ in their ability to appropriately infer kindness levels and speaker intent contained in literal exchanges. Notably, older adults answered more mental state questions 
assessing literal criticism comprehension correctly than younger adults. Finally, no agerelated impairments were found in terms of participants' factual understanding of the basic story line. Taken as a whole, the results indicate specific age-related deficits in making nonliteral interpretations of speech, rather than a more general problem in processing and remembering syntactic and semantic aspects of written language. Likewise, previous research demonstrated specific age effects on socio-cognitive abilities such as Theory of Mind (ToM) (e.g., Henry et al., 2013), emotion perception (Sullivan \& Ruffman, 2004), and sarcasm detection (Phillips et al., 2015). Age-related decline in ToM and emotion perception skills are related to difficulty in comprehending non-literal forms of speech (Mitchell \& Phillips, 2015; Shamay-Tsoory et al., 2015), indicating that the current results for interpreting the meaning underlying speech reflect a wider pattern of age differences in social cognition.

One possible explanation for our finding is age-related working memory decline (e.g., Wang et al., 2011). However, participants' memory for the basic story line was explicitly assessed via dedicated factual understanding questions, and the results clearly indicate that older participants were able to remember contextual information for all story types. Thus, it appears that rather than having forgotten the context preceding the target statement necessary to interpret it correctly, older adults failed to successfully integrate or combine the contextual information with the contrasting information contained in the target statement.

Another possible cognitive factor which might be important in age differences in interpreting conflicting information is inhibition. Previous research indicates that there are often age differences in inhibitory control (Kramer, Humphrey, Larish, \& Logan, 1994; West \& Alain, 2000). Understanding non-literal language may require inhibition of the literal meaning of a message and substitution with an alternative meaning derived from the context (McDonald \& Pearce, 1996; Schwoebel, Dews, Winner, \& Srinivas, 2000). An alternative perspective (e.g., direct access and constraint satisfaction models, Gibbs, 2001) suggests that 
preliminary processing of the nonpragmatic (literal) meaning of a non-literal remark is not necessary to understand it. Rather, consideration of different alternative interpretations is required so that one interpretation that corresponds with the available (linguistic and nonlinguistic) contextual information best can be selected (Constraint Satisfaction Model; Gibbs, 2001). We did not assess inhibitory control in the current study, but future studies should ideally incorporate measures of inhibitory control (e.g., the Simon Task; Van der Lubbe \& Verleger, 2002). This could also allow to evaluate the potential role of inhibition in nonliteral language comprehension in relation to competing psycholinguistic theories.

The age differences found in interpreting non-literal language may have implications for social interaction. Younger adults' response pattern in the current study accords with Dews' and Winner's (1995) ‘tinge hypothesis', suggesting that an important function of nonliteral language is to mute the evaluative meaning of a non-literal remark. Thus, sarcasm would be perceived as less confrontational than direct criticism and the emotional impact of a non-literal compliment would be less positive than if a literal compliment had been made. In the current study, younger adults showed exactly this pattern. However, particularly with regard to non-literal compliments, older adults did not appreciate this "muting function". Any potential socio-emotional benefits of using non-literal language are conditional upon the ability to understand the meaning underlying a statement. Hence, our findings suggest potential consequences for intergenerational communication, with older adults' reduced understanding of non-literal language acting as a potential barrier. Also, the shared development of non-literal communication can be important in building up rapport through informality and shared humour. The extent to which age differences in understanding nonliteral language may impact intergenerational communication and rapport building are topics for future research to explore. 
In the current study we investigated non-literal language comprehension exclusively via written short stories, which is the norm in this field. Understanding mental states from text is important in reading and enjoying fiction (e.g., De Vega, Diáz, \& León, 1997; Knitsch, 1988). However, other modalities are important. For example, speech or facial expressions aid non-literal language comprehension (Kreuz \& Roberts, 1995). Future studies should assess the understanding of non-literal language in different age groups using more contextually rich formats including prosodic and visual cues. This is particularly important given evidence that age differences in other social cognition skills such as emotion perception may be substantially reduced when multimodal stimuli are used (e.g., Hunter, Phillips \& MacPherson, 2010) and contextual information is provided (Noh \& Isaacowitz, 2013).

Another issue to consider is that in everyday interactions sarcastic exchanges may be generated, received or observed by an individual. However, in the present study, non-literal language comprehension was exclusively investigated from a third person perspective. Future studies should incorporate a first-person perspective. It would also be interesting to manipulate familiarity with the person making a non-literal remark, for example by using protagonists known to the participant (e.g., "your partner, a good friend"). Given that older adults may be more motivated to perform well on social cognition tasks when familiarity is increased (Zhang, Fung, Stanley, Isaacowitz, \& Ho, 2013), age differences in interpreting non-literal language might decrease if the protagonists are more familiar. Indeed, there is evidence that older adults use sarcasm with their close contacts in affectionate ways (Liptak, Tate, Flatt, Oakley \&, Lingler, 2014) and that they are more likely to disengage from negative personal comments than younger adults (Charles \& Carstensen, 2008).

Like most aging studies, the younger group in this study comprised mostly students taking part in exchange for course credit, while the older sample comprised of highly motivated self-selected voluntary participants. As indicated by gerontological research, 
voluntary research participation is associated with high cognitive functioning and social class (e.g., Lindenberger et al., 2001). Accordingly, our age groups did not differ in terms of selfrated general health or education. This may limit generalisability of the present results. However, in spite of the older adults' likely above-average health, cognitive functioning and motivation, they performed very much worse than the younger sample on non-literal short story comprehension.

Another important aspect of our sample to consider is that whilst the older group consisted almost entirely native English speakers, $60 \%$ of the younger group were non-native English speakers. Although research (Kim, 2014) suggests that individuals performing a language-based task in their second language are at a disadvantage, as all the younger nonnative English speakers were enrolled in Scottish higher education, a high command of the English language can be assumed. Also, despite this potential disadvantage to the younger participant group, we still found substantially poorer performance in the older group in interpreting non-literal language.

In order to test whether the number of non-native speakers may have biased our young adult sample we additionally tested another 39 younger participants (19 native and 20 nonnative speakers). In the full sample of younger adults across both samples native $(n=35)$ and non-native English speakers $(n=44)$ did not differ from each other on any measure of literal or non-literal language comprehension (see $t$-tests reported as Supplementary material 2). Further analyses indicated that both younger groups (native and non-native) differed significantly from the older adults. This indicates that in the current sample the pattern of results found was not influenced by the native tongue of the participants. 


\section{Conclusion}

The aim of the current study was to provide new insights into older and younger adults' understanding of positively and negatively valenced non-literal language. As predicted, older adults experienced specific difficulties understanding non-literally conveyed intentions whilst their understanding of literal statements remained intact. Both older and younger adults exhibited relatively poorer comprehension of non-literal compliments compared to criticisms, however, this difference was particularly pronounced in older adults. Younger adults' response pattern indicated appreciation of the 'muting function' of nonliteral remarks given that they perceived non-literal compliments and criticisms as neither particularly kind nor mean. In contrast, older adults showed no understanding of this potential communicative function, evaluating positively intended irony as mean and critical sarcasm as relatively kind. Future research should further examine the underlying reasons for the observed age-related differences in non-literal language comprehension as well as examine whether and how different modalities and perspectives may mediate age differences. Finally, research on the potential real-life implications such as intergenerational communication as well as cultural factors is needed to establish a fine-grained framework of how non-literal language is used and understood across the life-span and different societies.

\section{Acknowledgements:}

We are grateful for the input of Ruth Filik in designing the stimuli, and we would like to thank Isla Donaldson and Hannah McDonald for carrying out some of the testing.

Declarations of interest: none. This research did not receive any specific grant from funding agencies in the public, commercial, or not-for-profit sectors. 


\section{References}

Boylan, J., \& Katz, A. N. (2013). Ironic expression can simultaneously enhance and dilute perception of criticism. Discourse Processes, 50, 187-209.

Brown, P., \& Levinson, S. C. (1987). Politeness: Some universals in language usage (Vol. 4). Cambridge University Press.

Bruntsch, R., \& Ruch, W. (2017). Studying irony detection beyond ironic criticism: Let's include ironic praise. Frontiers in Psychology, 8:606.

Champagne, M., Seendy, J. L., \& Joanette, Y. (2006). Effect of aging on non-literal language treatment. Canadian Journal on Aging = La Revue Canadienne du Vieillissement, 25, $55-64$.

Charles, S. T., \& Carstensen, L. L. (2008). Unpleasant situations elicit different emotional responses in younger and older adults. Psychology and Aging, 23, 495-504.

Colston, H. L. (1997). Salting a wound or sugaring a pill: The pragmatic functions of ironic criticism. Discourse Processes, 23, 25-45.

De Vega, M., Diaz, J. M., \& León, I. (1997). To know or not to know: Comprehending protagonists' beliefs and their emotional consequences. Discourse Processes, 23, 169192.

Dews, S., Kaplan, J., \& Winner, E. (1995). Why not say it directly? The social functions of irony. Discourse Processes, 19, 347-367.

Dews, S., \& Winner, E. (1995). Muting the meaning a social function of irony. Metaphor and Symbol, 10, 3-19.

Filik, R., Brightman, E., Gathercole, C., \& Leuthold, H. (2017). The emotional impact of verbal irony: Eye-tracking evidence for a two-stage process. Journal of Memory and Language, 93, 193-202. 
Filik, R., Țurcan, A., Thompson, D., Harvey, N., Davies, H., \& Turner, A. (2016). Sarcasm and emoticons: Comprehension and emotional impact. The Quarterly Journal of Experimental Psychology, 69, 2130-2146.

Gibbs Jr, R. W. (2001). Evaluating contemporary models of figurative language understanding. Metaphor and Symbol, 16, 317-333.

Giora, R. (1997). Understanding figurative and literal language: The graded salience hypothesis. Cognitive Linguistics (includes Cognitive Linguistic Bibliography), 8, 183-206.

Glucksberg, S. (1995). Commentary on nonliteral language: Processing and use. Metaphor and Symbol, 10, 47-57.

Hancock, J. T., Dunham, P. J., \& Purdy, K. (2000). Children's comprehension of critical and complimentary forms of verbal irony. Journal of Cognition and Development, 1, 227248.

Harris, M., \& Pexman, P. M. (2003). Children's perceptions of the social functions of verbal irony. Discourse Processes, 36, 147-165.

Hartley, J. T., Stojack, C. C., Mushaney, T. J., Annon, T. A., \& Lee, D. W. (1994). Reading speed and prose memory in older and younger adults. Psychology and Aging, 9, 216223.

Henry, J. D., Phillips, L. H., Ruffman, T., \& Bailey, P. E. (2013). A meta-analytic review of age differences in theory of mind. Psychology and Aging, 28, 826-839.

Hunter, E. M., Phillips, L. H., \& MacPherson, S. E. (2010). Effects of age on cross-modal emotion perception. Psychology and Aging, 25, 779-787.

Kim, J. (2014). How Korean EFL learners understand sarcasm in L2 English. Journal of Pragmatics, 60, 193-206. 
Kramer, A. F., Humphrey, D. G., Larish, J. F., \& Logan, G. D. (1994). Aging and inhibition: beyond a unitary view of inhibitory processing in attention. Psychology and Aging, 9, 491-512.

Kreuz, R. J., \& Link, K. E. (2002). Asymmetries in the use of verbal irony. Journal of Language and Social Psychology, 21, 127-143.

Kreuz, R. J., \& Roberts, R. M. (1995). Two cues for verbal irony: Hyperbole and the ironic tone of voice. Metaphor and Symbol, 10, 21-31.

Langdon, R., Davies, M., \& Coltheart, M. (2002). Understanding minds and understanding communicated meanings in schizophrenia. Mind \& Language, 17, 68-104.

Lindenberger, U., Gilberg, R., Little, T. D., Nuthmann, R., Pötter, U., \& Baltes, P. B. (2001). Sample selectivity and generalizability of the results of the Berlin Aging Study. The Berlin Aging Study: Aging from 70 to 100. New York: Cambridge University Press.

Luong, G., \& Charles, S. T. (2014). Age differences in affective and cardiovascular responses to a negative social interaction: The role of goals, appraisals, and emotion regulation. Developmental Psychology, 50, 1919-1930.

McDonald, S., \& Pearce, S. (1996). Clinical insights into pragmatic theory: Frontal lobe deficits and sarcasm. Brain and Language, 53, 81-104.

Mitchell, R. L., \& Phillips, L. H. (2015). The overlapping relationship between emotion perception and theory of mind. Neuropsychologia, 70, 1-10.

Moran, J. R. (2013). Lifespan development: The effects of typical aging on theory of mind. Behavioural Brain Research, 237, 32-40.

Noh, S. R., \& Isaacowitz, D. M. (2013). Emotional faces in context: Age differences in recognition accuracy and scanning patterns. Emotion, 13, 238-249. 
Pexman, P. M., \& Olineck, K. M. (2002). Does sarcasm always sting? Investigating the impact of ironic insults and ironic compliments. Discourse Processes, 33, 199-217.

Phillips, L. H., Allen, R., Bull, R., Hering, A., Kliegel, M., \& Channon, S. (2015). Older adults have difficulty in decoding sarcasm. Developmental Psychology, 51, 18401852.

Raaijmakers, J. G., Schrijnemakers, J. M., \& Gremmen, F. (1999). How to deal with "the language-as-fixed-effect fallacy": Common misconceptions and alternative solutions. Journal of Memory and Language, 41, 416-426.

Preacher, K. J., \& Hayes, A. F. (2004). SPSS and SAS procedures for estimating indirect effects in simple mediation models. Behavior Research Methods, Instruments, \& Computers, 36, 717-731.

Premack, D., \& Woodruff, G. (1978). Does the chimpanzee have a theory of mind? Behavioral and Brain Sciences, 1, 515-526.

Reed, A. E., Chan, L., \& Mikels, J. A. (2014). Meta-analysis of the age-related positivity effect: age differences in preferences for positive over negative information. Psychology and Aging, 29, 1-15.

Roberts, R. M., \& Kreuz, R. J. (1994). Why do people use figurative language? Psychological Science, 5, 159-163.

Ruffman, T., Henry, J. D., Livingstone, V., \& Phillips, L. H. (2008). A meta-analytic review of emotion recognition and aging: Implications for neuropsychological models of aging. Neuroscience \& Biobehavioral Reviews, 32, 863-881.

Schwoebel, J., Dews, S., Winner, E., \& Srinivas, K. (2000). Obligatory processing of the literal meaning of ironic utterances: Further evidence. Metaphor and Symbol, 15, 4761. 
Shamay-Tsoory, S. G., Tomer, R., \& Aharon-Peretz, J. (2005). The neuroanatomical basis of understanding sarcasm and its relationship to social cognition. Neuropsychology, 19, 288-300.

Sullivan, S., \& Ruffman, T. (2004). Emotion recognition deficits in the elderly. International Journal of Neuroscience, 114, 403-432.

Sullivan, K., Winner, E., \& Hopfield, N. (1995). How children tell a lie from a joke: The role of second-order mental state attributions. British Journal of Developmental Psychology, 13, 191-204.

Uekermann, J., Channon, S., \& Daum, I. (2006). Humor processing, mentalizing, and executive function in normal aging. Journal of the International Neuropsychological Society, 12, 184-191.

Uekermann, J., Thoma, P., \& Daum, I. (2008). Proverb interpretation changes in aging. Brain and Cognition, 67, 51-57.

Van der Lubbe, R. H., \& Verleger, R. (2002). Aging and the Simon task. Psychophysiology, 39, 100-110.

Wang, M., Gamo, N.J., Yang, Y., Jin, L. E., Wang, X. J., Laubach, M., ... \& Arnsten, A. F. (2011). Neuronal basis of age-related working memory decline. Nature, 476, 210-213.

Watson, D., Clark, L. A., \& Tellegen, A. (1988). Development and validation of brief measures of positive and negative affect: the PANAS scales. Journal of Personality and Social Psychology, 54, 1063.

West, R., \& Alain, C. (2000). Age-related decline in inhibitory control contributes to the increased Stroop effect observed in older adults. Psychophysiology, 37, 179-189. 
Winner, E, \& Leekam, S. (1991). Distinguishing irony from deception: Understanding the speaker's second-order intention. British Journal of Developmental Psychology, 9, 257-270.

Zhang, X., Fung, H. H., Stanley, J. T., Isaacowitz, D. M., \& Ho, M. Y. (2013). Perspective taking in older age revisited: A motivational perspective. Developmental Psychology, 49, 1848-1858. 